\title{
Protective Effect of Phosphates and Fluoride on the Dissolution of Hydroxyapatite and Their Interactions with Saliva
}

\author{
Michele M. Manarelli ${ }^{a} \quad$ Juliano P. Pessan ${ }^{a} \quad$ Alberto C.B. Delbem ${ }^{a}$ \\ Jackeline G. Amaral $^{\mathrm{a}}$ Mayra F. Paiva ${ }^{\mathrm{a}}$ Michele E. Barbour ${ }^{\mathrm{b}}$ \\ ${ }^{a}$ School of Dentistry, São Paulo State University (UNESP), Araçatuba, Brazil; 'b School of Oral and Dental Sciences, \\ University of Bristol, Bristol, UK
}

\section{Keywords}

Fluoride $\cdot$ Hydroxyapatite $\cdot$ Pellicle $\cdot$ Polyphosphates $\cdot$ Saliva

\begin{abstract}
This study aimed to investigate the effect of phosphates and fluoride, alone or in combination, and the influence of salivary pellicle on hydroxyapatite $(\mathrm{HA})$ dissolution. The baseline dissolution rate of $\mathrm{HA}$ discs was measured using a $\mathrm{pH}$ stat system ( $0.3 \%$ citric acid, $\mathrm{pH} 3.2)$. In the first series of experiments, HA discs ( $n=8 /$ group) were treated with: a placebo solution (PLA, deionised water); sodium trimetaphosphate (TMP), sodium tripolyphosphate (TRI) and sodium pyrophosphate (PYRO) at 1 or $8 \% ; 500$ ppm F; 1,100 ppm F; 1,100 ppm F/1\% TMP; 1,100 ppm F/8\% TMP; 1,100 ppm F/1\% TRI; 1,100 ppm F/8\% TRI. In the second phase, HA discs were immersed in pooled human saliva $\left(37^{\circ} \mathrm{C} / 2 \mathrm{~h}\right)$ and treated with PLA, 1,100 ppm F/1\% TMP, 1,100 ppm F/8\% TMP, 1,100 ppm F/1\% TRI, and 1,100 ppm F/8\% TRI. After treatments, final dissolution rates were measured from 3 consecutive 30 min assays. Statistical analyses were performed using 2-way ANOVA followed by the Fisher test $(a=0.05)$. The type and concentration of phosphate tested significantly influenced HA dissolution; 8\% TRI showed the highest reduction (36.9\%) among all treatment solutions. Fluoride alone (1,100 ppm F)
\end{abstract}

significantly reduced HA dissolution by $20.7 \%$. When fluoride and phosphates were associated, 1,100 ppm F/1\% TMP, $1,100 \mathrm{ppm} F / 8 \% \mathrm{TMP}$, and 1,100 ppm F/8\% TRI showed the highest percentage reductions of dissolution (40.3-46.1\%). Salivary pellicle led to a greater and more sustained protective effect of the treatment solutions compared to their counterparts without salivary coating. It was concluded that the association of phosphate and fluoride enhanced their protective effect against HA dissolution when compared with these compounds alone, especially in the presence of salivary pellicle.

(c) 2017 S. Karger AG, Basel

Dental erosion is defined as the softening of the tooth surface followed by its gradual bulk removal, caused by exposure to acids of non-bacterial origin [Lussi et al., 2011]. Surface softening and enamel loss are most often caused by the consumption of acidic soft drinks containing citric and/or phosphoric acids [Zero and Lussi, 2005].

Conventional fluoride preparations such as toothpastes or mouth rinses have a limited effect on erosion [Wiegand and Attin, 2003; Magalhães et al., 2011], and significant inhibition requires either the application in high concentrations or at high frequency [Ganss et al.,

\section{KARGER}

(c) 2017 S. Karger AG, Basel

E-Mail karger@karger.com

www.karger.com/cre
Prof. Juliano P. Pessan

Department of Pediatric Dentistry and Public Health School of Dentistry, São Paulo State University (UNESP)

Rua José Bonifácio 1193, Araçatuba, SP 16015-050 (Brazil)

E-Mail jpessan@foa.unesp.br 
2004], or the use of preparations containing titanium or stannous ions [Ganss et al., 2010; Wiegand et al., 2010] that might be unsuitable for routine use because of their low $\mathrm{pH}$ and their propensity to stain the teeth. Nonetheless, promising results have been described for fluoridated products associated with sodium trimetaphosphate on erosion in vitro [Moretto et al., 2010; Manarelli et al., 2011, 2013] and in situ [Moretto et al., 2013]. Similarly, sodium tripolyphosphate and sodium pyrophosphate tetrabasic have also been shown to promote significant reductions on the dissolution of HA discs [Barbour et al., 2005] and HA crystals [Scaramucci et al., 2011, 2015] using a $\mathrm{pH}$-stat approach, despite these phosphates not being able to reduce enamel and dentine wear in vitro [Scaramucci et al., 2011].

Saliva contains phosphates, proteins and bicarbonate buffers and is supersaturated with respect to tooth minerals, such as calcium and phosphate. It is known that proteins can protect the teeth by the formation of a salivary pellicle when teeth are exposed to saliva [Siqueira et al., 2007]. This pellicle of adsorbed salivary proteins might act as a diffusion barrier or a selective permeable membrane reducing direct contact between acids and tooth surface and thus reducing demineralization of the surface [Wetton et al., 2007; Jager et al., 2011; White et al., 2012].

Based on the above, the aim of the present study was to investigate the effect of fluoride and phosphates, alone or in combination, on the dissolution of hydroxyapatite (HA), as well as the influence of the salivary pellicle on this process. The study hypothesis was that HA dissolution would be significantly reduced by the presence of fluoride and phosphates, and that this effect would be influenced by the presence of salivary acquired pellicle.

\section{Materials and Methods}

Materials

Discs of compressed HA, mean diameter $12.1 \mathrm{~mm}$, mean thickness $1.23 \mathrm{~mm}$, were purchased from HiMed Inc., Old Bethpage, NY, USA. All products were obtained from Sigma-Aldrich (Poole, UK). Solutions were prepared using deionised water.

\section{Groups}

Treatment solutions included: placebo (PLA, deionised water); sodium trimetaphosphate at 1 and 8\% (1\% TMP and 8\% TMP, respectively); sodium tripolyphosphate at 1 and $8 \%$ (1\% TRI and $8 \%$ TRI, respectively); sodium pyrophosphate tetrabasic at 1 and $8 \%$ (1\% PYRO and 8\% PYRO, respectively); fluoride (500 ppm F and $1,100 \mathrm{ppm} F$, as sodium fluoride); $1,100 \mathrm{ppm} F$ with TMP at 1 and $8 \%(1,100 \mathrm{ppm}$ F/1\% TMP and 1,100 ppm F/8\% TMP, respectively); 1,100 ppm F with TRI at 1 and $8 \%(1,100 \mathrm{ppm} \mathrm{F/1 \%} \mathrm{TRI} \mathrm{and}$
1,100 ppm F/8\% TRI). Fluoride concentrations were chosen based on those present in conventional formulations (1,100 ppm F), as well as in low-fluoride toothpastes (500 ppm F) available in some countries.

\section{Measurement of Dissolution Rate}

Dissolution in $0.3 \%$ citric acid, $\mathrm{pH} 3.2$, was measured using a pH-stat model (718 Stat Titrino: Metrohm UK, Runcorn, UK) with a $50-\mathrm{mL}$ double-walled glass reaction vessel fitted with a lid with 3 inlet ports. Water was pumped by a circulating water bath (type GD120; Grant Instruments, Cambridge, UK) through the water jacket to maintain the reaction temperature at $37^{\circ} \mathrm{C}$. Prior to use in the $\mathrm{pH}$-stat, batches of discs were conditioned to ensure consistency of response. The discs were first ultrasonicated in deionised water in a bath with an ultrasonic power of $40 \mathrm{kHz}$ for 15 min to remove loose HA particles. They were then exposed to gently stirred $0.3 \%$ citric acid, $\mathrm{pH} 3.2$, for $30 \mathrm{~min}$ at room temperature, then washed in deionised water and finally air-dried. For use in the $\mathrm{pH}$-stat, the backs of the discs were coated with nail varnish to leave an area of $161.4 \mathrm{~mm}^{2}$ available for reaction. Discs were then fixed with sticky wax to the tip of glass tubes fitted with a cone suitable for the inlet ports.

In each experiment, $30 \mathrm{~mL}$ of citric acid solution was introduced into the reaction vessel and the $\mathrm{pH}$ electrode and burette tip fitted. After the system had reached thermal equilibrium, the $\mathrm{pH}$ was adjusted to 3.2 by adding small quantities of concentrated $\mathrm{KOH}$ or $\mathrm{HCl}$ solution and finally adjusted using the $\mathrm{pH}$-stat. The reaction was initiated by introducing the specimen on its holder and addition of titrant $(50 \mathrm{mM} \mathrm{HCl})$ was recorded for $30 \mathrm{~min}$. A control measurement of dissolution rate was made after the conditioning step, and the disc was removed from the holder, washed in deionised water and dried. After exposing the disc to the chosen treatment, the disc was reattached to the glass specimen holder, and measurement of the dissolution rate was made.

\section{Saliva Collection}

Mixed saliva was collected from a panel of 2 volunteers, who had previously registered at the saliva bank from the University of Bristol, having received local ethical approval for this. When saliva was required, each volunteer was provided with a $50-\mathrm{mL}$ polystyrene universal tube, marked at the $25-\mathrm{mL}$ level. Each volunteer chewed a square of Parafilm to stimulate salivary flow and expectorated saliva into the tube until the mark was reached. These samples were combined and centrifuged using a Centaur 1 (MSE, London, $\mathrm{UK}$ ) at $4,000 \mathrm{~g}$ for $15 \mathrm{~min}$ at ambient temperature. The supernatant was used to treat HA specimens $(2 \mathrm{~mL} /$ disc $)$.

\section{Experiments}

Native HA

In the first series of experiments, inhibition was tested on nonsaliva-treated HA [Barbour et al., 2005; Jones et al., 2013]. First, a sequence of 3 control measurements was performed. Next, the HA discs were individually immersed in $100 \mathrm{~mL}$ of the treatment solutions (PLA; 1\% TMP; $8 \%$ TMP; 1\% TRI; $8 \%$ TRI; 1\% PYRO; $8 \%$ PYRO; 500 ppm F; 1,100 ppm F; 1,100 ppm F/1\% TMP; 1,100 ppm F/8\% TMP; 1,100 ppm F/1\% TRI; 1,100 ppm F/8\% TRI) for 2 min with gentle stirring, followed by rinsing with deionised water. Following this, the posttreatment dissolution rate was determined in 3 consecutive periods of $30 \mathrm{~min}$ each. For this, $30 \mathrm{~mL}$ of citric acid was added to the reaction vessel and the $\mathrm{pH}$ electrode and burette 
tip were fitted. After the system had reached equilibrium ( $\mathrm{pH} 3.2$ adjusted with $\mathrm{KOH}$ or $\mathrm{HCl}$ ), the reaction was initiated by introducing the $\mathrm{HA}$ disc, and the addition of titrant $(50 \mathrm{mM} \mathrm{HCl})$ was recorded for $30 \mathrm{~min}$ using $\mathrm{pH}$-stat, similarly as done for the control. Fresh citric acid solutions were added to the vessel for each 30 -min assay. This sequence was performed on 8 separate HA discs for each treatment.

\section{Saliva-Coated HA}

After making control measurements of dissolution rate, discs were immersed in pooled mixed saliva supernatant for $2 \mathrm{~h}$ in Petri dishes in an incubator at $37^{\circ} \mathrm{C}$. Next, the HA discs were immersed in treatment solution (PLA; $1,100 \mathrm{ppm}$ F/1\% TMP; $1,100 \mathrm{ppm}$ F/8\% TMP; 1,100 ppm F/1\% TRI; 1,100 ppm F/8\% TRI) 100 mL for 2 min with gentle stirring. After rinsing in water, a series of 3 posttreatment measurements of dissolution was performed. This sequence was performed on 8 separate HA discs for each treatment.

\section{Statistical Analysis}

Analyses were performed using the SigmaPlot (version 12.0) with $5 \%$ of statistical significance level. Data from native and saliva-coated discs exhibited a normal (Kolmogorov-Smirnov) and homogeneous distribution. Treatment solutions (with or without salivary pellicle) and time (baseline control, posttreatment 1, 2, and 3) were considered as variation factors. The baseline was determined for each specimen by averaging the 3 control runs before treatment. Thus, data were submitted to 2-way ANOVA, followed by the Fisher test $(p<0.05)$.

\section{Results}

Ten of the test solutions without pellicle effected a statistically significant reduction in the HA dissolution rate in citric acid solutions (Table 1). Among the phosphates, the highest percentage reduction of dissolution was seen for $8 \%$ TRI (36.9\%), while 1,100 ppm F had the highest percentage reduction of dissolution (20.7\%) among the solutions containing only NaF. For solutions containing $\mathrm{NaF}$ and a phosphate salt in combination, 1,100 ppm F/1\% TMP, 1,100 ppm F/8\% TMP and 1,100 ppm F/8\% TRI showed the highest percentage reductions of dissolution (Table 1), ranging from 40.3 to $46.1 \%$.

Regarding the presence of salivary pellicle, all test solutions effected a statistically significant reduction in the HA dissolution rate in citric acid solution in specimens covered with salivary pellicle in comparison with their counterparts not treated with saliva (Fig. 1). Salivary coating promoted a more sustained protective effect of the treatment solutions when compared to their counterparts not treated with saliva $(p<0.001)$. Also, no significant differences were seen for the treatment groups in the presence of saliva regarding the reduction of dissolution rate and persistence of effect over time.
Table 1. Mean (and SD) percentage reduction of HA dissolution for each treatment solution after exposure to citric acid according to time following exposure to treatment solutions

\begin{tabular}{|c|c|c|c|}
\hline Groups & $\begin{array}{l}\text { Post- } \\
\text { treatment run } 1 \\
(30 \mathrm{~min})\end{array}$ & $\begin{array}{l}\text { Post- } \\
\text { treatment run } 2 \\
(60 \mathrm{~min})\end{array}$ & $\begin{array}{l}\text { Post- } \\
\text { treatment run } 3 \\
(90 \mathrm{~min})\end{array}$ \\
\hline PLA & $0.7^{\mathrm{a}}(2.1)$ & $0.6^{\mathrm{a}}(2.1)$ & $0.0^{\mathrm{a}}(1.3)$ \\
\hline $1 \% \mathrm{TMP}$ & $1.2^{\mathrm{a}}(5.8)$ & $0.4^{\mathrm{a}}(4.6)$ & $0.0^{\mathrm{a}, \mathrm{e}}(3.8)$ \\
\hline $8 \%$ TMP & $16.2^{\mathrm{b}, \mathrm{c}}(4.4)^{*}$ & $10.3^{\mathrm{b}, \mathrm{f}}(3.6)$ & $5.2^{\mathrm{b}}(5.4)$ \\
\hline $1 \% \mathrm{TRI}$ & $12.6^{\mathrm{b}}(4.2)^{*}$ & $8.4^{\mathrm{b}, \mathrm{f}}(3.7)$ & $2.0^{\mathrm{b}, \mathrm{e}}(3.8)$ \\
\hline $8 \%$ TRI & $36.9^{\mathrm{c}, \mathrm{d}}(5.4)^{*}$ & $25.7^{c}(3.6)^{*}$ & $17.1^{\mathrm{c}}(7.5)^{*}$ \\
\hline $1 \%$ PYRO & $7.7^{\mathrm{a}, \mathrm{b}}(6.7)$ & $3.6^{\mathrm{b}}(5.8)$ & $0.0^{\mathrm{b}}(5.2)$ \\
\hline 8\% PYRO & $18.2^{\mathrm{b}, \mathrm{c}}(4.9)^{*}$ & $10.3^{\mathrm{b}, \mathrm{d}}(2.8)^{*}$ & $4.6^{\mathrm{b}}(2.5)$ \\
\hline 500 ppm F & $13.1^{\mathrm{b}}(3.3)^{*}$ & $9.0^{\mathrm{d}, \mathrm{f}}(4.2)^{*}$ & $4.8^{\mathrm{b}}(6.1)$ \\
\hline $1,100 \mathrm{ppm} \mathrm{F}$ & $20.7^{c}(5.7)^{*}$ & $12.3^{\mathrm{b}}(1.7)^{*}$ & $8.5^{\mathrm{b}}(4.0)$ \\
\hline $1,100 \mathrm{ppm} \mathrm{F} / 1 \% \mathrm{TMP}$ & $40.3^{\mathrm{d}}(5.5)^{*}$ & $17.2^{\mathrm{e}}(4.7)^{*}$ & $2.6^{\mathrm{d}}(5.8)$ \\
\hline $1,100 \mathrm{ppm} F / 8 \%$ TMP & $42.2^{\mathrm{d}}(4.8)^{*}$ & $13.1^{\mathrm{e}}(6.5)^{*}$ & $4.6^{\mathrm{d}}(5.8)$ \\
\hline 1,100 ppm F/1\% TRI & $32.3^{\mathrm{c}, \mathrm{d}}(7.8)^{*}$ & $11.5^{\mathrm{b}, \mathrm{d}}(8.1)^{*}$ & $7.0^{\mathrm{b}}(7.5)$ \\
\hline $1,100 \mathrm{ppm} \mathrm{F} / 8 \%$ TRI & $46.1^{\mathrm{d}}(3.3)^{*}$ & $16.9^{f}(6.8)^{*}$ & $5.4^{\mathrm{b}}(6.2)$ \\
\hline
\end{tabular}

Different superscript letters within each column show statistical differences among treatment solutions. Asterisks indicate a significant difference from mean baseline control (Fisher test, $p<0.05, n=8$ /group). PLA, placebo solution (deionised water); TMP, sodium trimetaphosphate; TRI, sodium tripolyphosphate; PYRO, sodium pyrophosphate.

\section{Discussion}

In this study, the $\mathrm{pH}$-stat method was employed to investigate the immediate and sustained effect of TMP, TRI and PYRO with or without fluoride on HA dissolution. These phosphates have shown promising results against dental erosion, but most studies only evaluated their immediate effects. The present study showed that the reduction of HA dissolution as well as the duration of this effect was significantly influenced by the type of phosphate and the concentration tested, as well as by the salivary coating. Therefore, the study's hypothesis was accepted.

In the first set of experiments, all test solutions were evaluated without the presence of a salivary coating. It has been shown that fluoride can offer a protection against dental erosion and this effect is given by a formation of a layer of $\mathrm{KOH}$-soluble calcium fluoride [Magalhães et al., 2011]. In the present study, a relationship between fluoride concentration and the protective effect was observed, since reductions in HA dissolution were 13 and $21 \%$, respectively, for solutions containing 500 and 1,100 ppm F. The results are in line with previous data showing a reduction of $12 \%$ in dissolution rate of native HA when 300 ppm F was administered [Jones et al., 2013]. However, these reductions did not persist beyond the first posttreat-
98

Caries Res 2017;51:96-101 DOI: $10.1159 / 000452716$
Manarelli/Pessan/Delbem/Amaral/Paiva/ Barbour 
Fig. 1. Mean inhibition of HA dissolution for each treatment solution after exposure to citric acid in specimens with or without salivary coating. Bars indicate standard deviations. Asterisks indicate significant differences between each posttreatment run and the control (baseline) measurement. Different superscript letters indicate significant differences among the groups (Fisher test, $p<0.05, n=8$ /group).

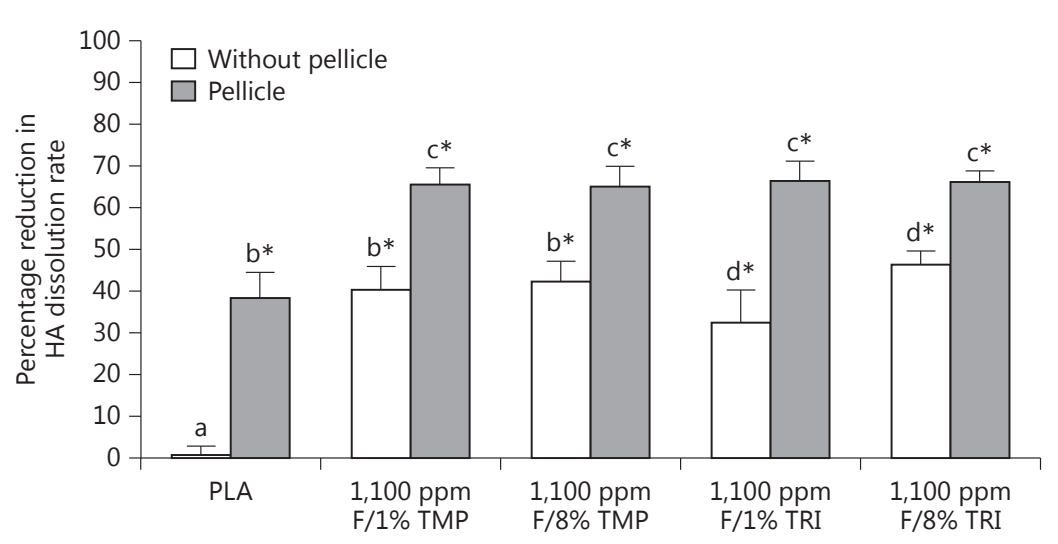

a

Posttreatment run 1 (30 $\mathrm{min}$ )

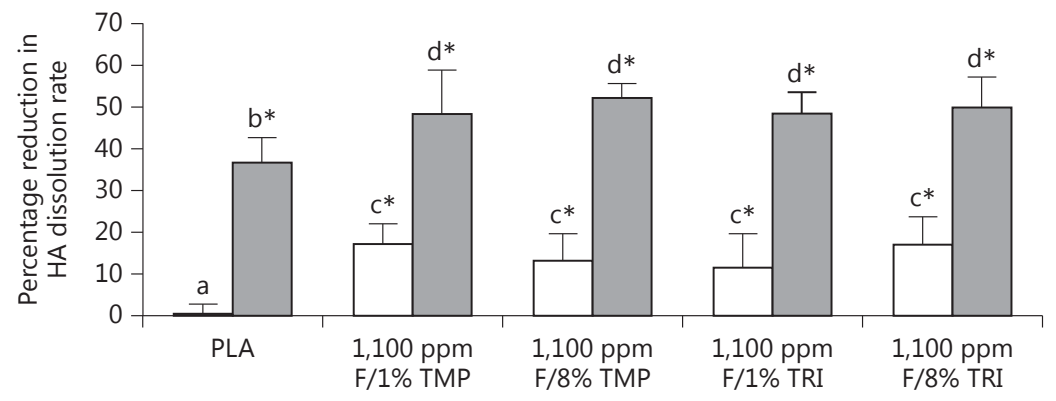

b

Posttreatment run 2 (60 min)

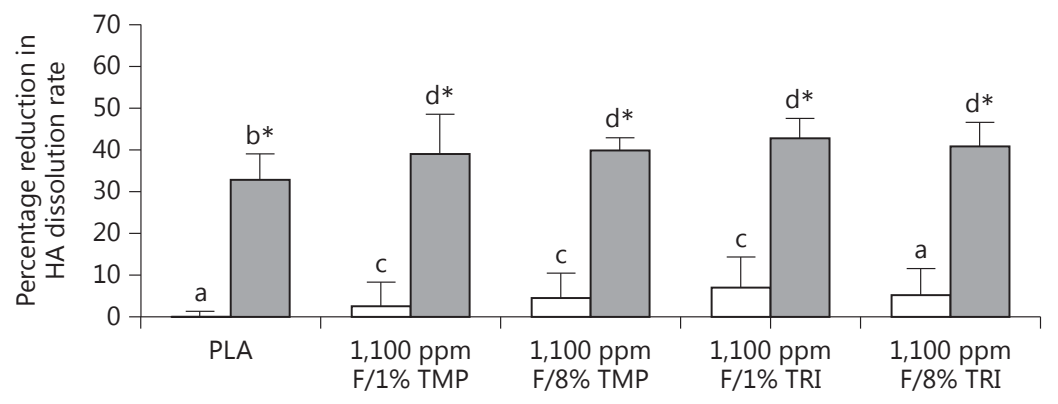

C

Posttreatment run 3 (90 min) ment run, confirming that fluoride alone might have a limited action against enamel erosion.

Regarding the phosphates evaluated, the highest reduction of HA dissolution rate (approx. 37\%) was observed for the solution containing $8 \%$ of TRI, and its effects were persistent up to the third posttreatment run (90 min). Despite the fact that the present results are higher than data reported in previous investigations using the same methodology [Barbour et al., 2005; Scaramucci et al., 2011; 2015], it is noteworthy that the studies cited above used lower concentrations of this phosphate (ranging from 0.02 to $2 \%$ ), which might help to explain the different results. This assumption is reinforced by the fact that $1 \%$ TRI promoted a much lower reduction in HA dissolution (approx. $13 \%)$ than at $8 \%$, and its effects were only significant for the first posttreatment run $(30 \mathrm{~min})$. As for the other phosphates tested, the reductions in HA dissolution were less pronounced for TMP and PYRO at $8 \%$, and their effects did not persist over the 3 posttreatment runs. The modest effect of TMP is in agreement with previous find- 
ings showing that TMP alone produces negligible effects on bovine enamel de-/remineralisation when added to different topically applied dental products [Danelon et al., 2014; Manarelli et al., 2014]. The association of TMP and TRI with fluoride further reduced the rate of HA dissolution compared to these phosphates or fluoride alone. It is noticeable that while $1 \%$ TMP did not produce any significant effect on HA dissolution (1.2\%), its association with $1100 \mathrm{ppm} F$ led to a maximum inhibition of $40.3 \%$. Given that this value was 2-fold higher than that observed for $1100 \mathrm{ppm} F$ alone, the results indicate that TMP and fluoride have a synergistic effect against HA dissolution, confirming previous observations using bovine enamel in a pH-cycling model [Castro et al., 2015]. In this sense, although TMP has been shown to act like a partial barrier to $\mathrm{CaF}_{2}$ deposition on enamel surface [Manarelli et al., 2014], $\mathrm{CaF}_{2}$ and $\mathrm{CaF}^{+}$compounds are believed to remain on TMP molecules (adhered to HA) and to be released to saliva upon acidification of the oral environment, further reacting with salivary phosphates leading to the formation of more reactive calcium phosphates [Manarelli et al., 2014]. It is also noteworthy that the association with fluoride led to a significant increase in the protective effect of TMP at both concentrations tested (1 and $8 \%$ ), while for TRI this effect was only seen at $1 \%$, indicating that each phosphate has an ideal molar ratio with fluoride in order to achieve the maximum additive or synergistic effect.

In the second set of experiments, a salivary coating on HA was introduced, as it is known that saliva plays an important role on dental erosion. The acquired pellicle is composed of proteins and glycoproteins that act as a protective barrier, preventing the direct contact between the acid and the tooth surface [Buzalaf et al., 2012]. When compounds were tested with a salivary coating, a greater reduction of HA dissolution (approx. 65\% for all solutions together) was observed compared with discs not previously treated with saliva (approx. 40\% for all solutions together) in the first posttreatment run, which is also in line with previous data using a similar research protocol [Jones et al., 2013]. It is noteworthy that while the protective effect for native HA decreased by $63 \%$ between the first and the second posttreatment run, and by $66 \%$ between the second and the third posttreatment run, these decreases were much lower for HA previously exposed to saliva (15 and $20 \%$, respectively). This indicates that the protective layer formed by salivary components, fluoride and TMP or TRI were not totally dissolved from the HA surface after the first exposure to the acid medium but occurred gradually from its external to the basal components [Joiner et al., 2008].
Although the present in vitro model does not fully reproduce acid challenges occurring in vivo, our data can be helpful in further investigations. In this sense, the results indicate that fluoride associated with phosphates such as TRI and TMP could be an alternative to the development of oral products against dental erosion. It is important to highlight that an optimal ratio between phosphate and fluoride should be used in order to achieve optimum results, based on the present results and also on previous data from experiments performed with bovine enamel specimens [Takeshita et al., 2009; Castro et al., 2015]. Another important point is related to the presence of saliva in future studies, as the effect of the treatments was shown to be highly dependent on the salivary coating of the specimens [Buzalaf et al., 2012]. Thus, the screening of such agents should always include specimens that have been pretreated with saliva as well as native surfaces.

To sum up, it can be concluded that TMP and TRI provided reduction of HA dissolution when an erosion-like model was used. In addition, the association of these phosphates with fluoride enhanced their effectiveness compared to fluoride or phosphates alone. Thus, these associations could be a potential alternative in future investigations in order to prevent dental erosion.

\section{Acknowledgements}

The authors thank the CAPES for the PhD scholarship (process BEX 9221-12-3) to the first author.

\section{Disclosure Statement}

The authors declare no conflict of interest that might introduce bias or affect the manuscript's judgment.

\section{Author Contributions}

Study idea and design: J.P.P., A.C.B.D., and M.E.B., Accomplishment of experiments: M.M.M., J.G.A., and M.E.B. Data analysis: J.G.A., M.F.P., J.P.P., and A.C.B.D. Manuscript preparation: M.M.M., J.P.P., A.C.B.D., J.G.A., M.F.P., and M.E.B. 


\section{References}

Barbour ME, Parker DM, Allen GC, Jandt KD: Human enamel erosion in constant composition citric acid solutions as a function of degree of saturation with respect to hydroxyapatite. J Oral Rehabil 2005;32:16-21.

Buzalaf MAR, Hannas AR, Kato MT: Saliva and dental erosion. J Appl Oral Sci 2012;20:493502.

Castro LP, Delbem ACB, Danelon M, Passarinho A, Percinoto C: In vitro effect of sodium trimetaphosphate additives to conventional toothpastes on enamel demineralization. Clin Oral Investig 2015;19:1683-1687.

Danelon M, Takeshita EM, Peixoto LC, Sassaki KT, Delbem AC: Effect of fluoride gels supplemented with sodium trimetaphosphate in reducing demineralization. Clin Oral Investig 2014;18:1119-1127.

Ganss C, Klimek J, Brune V, Schürmann A: Effects of two fluoridation measures on erosion progression in human enamel and dentine in situ. Caries Res 2004;38:561-566.

Ganss C, Neutard L, von Hinckeldey J, Klimek J, Schlueter N: Efficacy of a tin/fluoride rinse: a randomized in situ trial on erosion. J Dent Res 2010;89:1214-1218.

Jager DHJ, Vieira AM, Ligtenberg AJM, Bronkhorst EM, Huysmans MCDNJM, Vissink A: The effect of saliva on the susceptibility of hydroxyapatite to erosion. Caries Res 2011; 45:532-537.

Joiner A, Schwarz A, Philpotts CJ, Cox TF, Huber $\mathrm{K}$, Hannig M: The protective nature of pellicle towards toothpaste abrasion on enamel and dentine. J Dent 2008;36:360-368.

Jones SB, Rees GD, Shellis RP, Barbour ME: The effect of monoalkyl phosphates and fluoride on dissolution of hydroxyapatite, and interactions with saliva. Caries Res 2013;47:355-363.

Lussi A, Schlueter N, Rakhmatullina E, Ganss C: Dental erosion - an overview with emphasis on chemical and histopathological aspects. Caries Res 2011;45:33-42.

Magalhães AC, Wiegand A, Rios D, Buzalaf MA, Lussi A: Fluoride in dental erosion; in Buzalaf MA (ed): Fluoride in the Oral Cavity. Monogr Oral Sci. Basel, Karger, 2011.

Manarelli MM, Delbem AC, Lima TM, Castilho FC, Pessan JP: In vitro remineralizing effect of fluoride varnishes containing sodium trimetaphosphate. Caries Res 2014;48:299305.

Manarelli MM, Moretto MJ, Sassaki KT, Martinhon CC, Pessan JP, Delbem AC: Effect of fluoride varnish supplemented with sodium trimetaphosphate on enamel erosion and abrasion. Am J Dent 2013;26:307-312.

Manarelli MM, Vieira AEM, Matheus AA, Sassaki KT, Delbem ACB: Effect of mouth rinses with fluoride and trimetaphosphate on enamel erosion: an in vitro study. Caries Res 2011; 45:506-509.

Moretto MJ, Delbem AC, Manarelli MM, Pessan JP, Martinhon CC: Effect of fluoride varnish supplemented with sodium trimetaphosphate on enamel erosion and abrasion: an in situ/ex vivo study. J Dent 2013;41:1302-1306.

Moretto MJ, Magalhães AC, Sassaki KT, Delbem AC, Martinhon CC: Effect of different fluoride concentrations of experimental dentifrices on enamel erosion and abrasion. Caries Res 2010;44:135-140.

Scaramucci T, Borges AB, Lippert F, Zero DT, Aoki IV, Hara AT: Anti-erosive properties of solutions containing fluoride and different film-forming agents. J Dent 2015;43:458-465.

Scaramucci T, Hara AT, Zero DT, Ferreira SS, Aoki IV, Sobral MA: In vitro evaluation of the erosive potential of orange juice modified by food additives in enamel and dentine. J Dent 2011;39:841-848.

Siqueira WL, Zhang W, Helmerhorst EJ, Gygi SP, Oppenheim FG: Identification of protein components in in vivo human acquired enamel pellicle using LC-ESI-MS/MS. J Proteome Res 2007;6:2152-2160.

Takeshita EM, Castro LP, Danelon M, Sassaki KT, Delbem AC: In situ evaluation of dentifrice with low fluoride content and supplemented with trimetaphosphate. Caries Res 2009;43: 219-222.

Wetton S, Hughes J, Newcombe RG, Addy M: The effect of saliva derived from different individuals on the erosion of enamel and dentine: a study in vitro. Caries Res 2007;41:323327

White AJ, Jones SB, Barbour ME, Churchley DR, Gracia LH, Rees GD: Inhibition of erosive dissolution by sodium fluoride: evidence for a dose-response. J Dent 2012;40:654-660.

Wiegand A, Attin T: Influence of fluoride on the prevention of erosive lesions - a review. Oral Health Prev Dent 2003; 1:245-253.

Wiegand A, Magalhães AC, Attin T: Is titanium tetrafluoride $\left(\mathrm{TiF}_{4}\right)$ effective to prevent carious and erosive lesions? A review of the literature. Oral Health Prev Dent 2010;8:159164.

Zero DT, Lussi A: Erosion-chemical and biological factors of importance to the dental practitioner. Int Dent J 2005;55:285-290. 